\title{
HOW TO INTERPRET CODES
}

A. TYPES OF CODES.In addition to the abbreviations used in the English gloss section (see List of Abbreviations below), there are two sorts of codes used in this work: word-class codes, and derivative-type codes.

Word-class codes classify single words (but not compounds or idioms) according to their Nukuoro grammatical category. A list of these is presented below. (See Appendix 4 for word-class definitions.)

Derivative-type codes classify words and phrases according to their relationship to a base form (the form in a series of related forms which is closest in form to the root). Derivative-type codes are written in three columns:the symbol in the first column indicates the type of stem; the symbol in the second column indicates the prefix, and the symbol in the third column indicates the type of suffix. A summary list is presented below. (Further information on the meaning of these distinctions is contained in Appendix 1.)

\section{B. SUMMARY OF DERIVATIVE-TYPE CODES}

\section{Col. 1: Stem types}

B Base form

C Variant of a base form

E Emphatic form

$P$ Plural concord form

R Reduplicated form

$S$ Second reduplicated form

T Partially reduplicated form

$\mathrm{X}$ Compound word

(If col. 1 is blank refer to the section on Additional forms below.) 
Subtypes XCO compound word

$\mathrm{XE} \mathrm{XCO}+$ the prefix haga- (see below

$$
\text { under Prefixes) }
$$

XFL name of an animal or plant species

XG name of an item of native manufacture

$\mathrm{U}$ Unclassified

Col. 2: Prefixes
C $m a-$
Resultative Prefix
D he-
Reciprocal Prefix
E haga-
Causative Prefix
F haga- +ma-
(E+C, above)
G haga-the-
(E+D, above)

(A blank in this column means that the stem is uninflected.)

Col. 3: Suffixes
1 -nga
Nominal SuffiX
$2-a$
Perfective Suffix
$3-a+n g a$

(A blank in this column means that the stem is uninflected.) Additional forms

ID An idiom

CF Compare the above with the following form, which may be related to this root.

C. WORD-CLASS CODES
A Adjective
AN Numeral
B Base surrogate
D Dependent word
I Interjection
M ... Minor Morpheme
MA Article
MC Numeral Classifier
MF Prefix
MI Introductory Word
MP Preposition
MR Relational Particle
MT Tense-aspect Marker
MV Adverb
MZ Suffix
N Noun 
Nukuoro Lexicon

NA Adjectival Noun

NL Location Word

NN Name of a Person

NP Place Name

NT Time Noun

P... Pronoun

PI Independent Pronoun

PP ... Possessive Pronoun

PD. . . Dependent Pronoun

PT ... Distributive Pronoun

U Unclassified

V Verb 